there having become imperceptible at the wrist. Caffeine, strychnine, and brandy were administered hypodermically. The patient recovered consciousness sufficiently to ask for a drink and to say that she felt as if she were dying. At 1.30 P.M. she died, just two hours after the anæsthetic had been stopped. Unfortunately no post-mortem was obtained.

It seems likely that this patient was suffering from some form of myocarditis. The case demonstrates how useless the ordinary examination of the heart with a stethoscope may be as a precaution taken before an anæsthetic is administered.

CASE 3.-A healthy-looking baby, 7 months old, suffering with harelip., Anæsthesia was commenced with chloroform on a Junker's mask. Before full surgical anæesthesia was after the substitution the patient suddenly grew pale, the breathing became very shallow, the pupils dilated, and there was complete muscular relaxation. The anæsthetic was stopped, the head lowered, and the face rubbed with a cloth. After some minutes recovery took place, the breathing becoming deeper and the colour much better. The ether was not reapplied unut his eyes. A catheter was introduced into one nostril and light anæesthesia maintained by means of Kelly's insufflation apparatus. At the end of an hour, however, the respirations, which had been regular, were noticed to stop, the colour becoming pale and the pupils dilating. In spite of everything that could be done-e.g., artificial respiration, administration of oxygen, massage of the heart through the chest wall - the child failed to respond. There was no post-mortem.

It seems likely that this child may have been one of those cases of status lymphaticus which are so likely to die during anæsthesia. Having seen many cases of pallor with faint breathing and complete muscular relaxation in babies under the influence of ether, where chloroform was used for induction, but had been discontinued a few minutes before, we have completely abandoned the use of chloroform at the Royal Victoria Hospital for this purpose and now never see the condition.

CASE 4.-A well-developed, muscular lumberman of 50 , suffering from a slowly growing tumour of the right femur. His lungs were normal. The heart dullness extended above to the third rib and on the left to $4 \frac{1}{2}$ inches from the midsternal line. The heart sounds were distant but no murmurs were heard. He was given a preliminary hypodermic injection of morphine gr. 1 and atropine gr. 1/150. Ether by the open method was commenced at 1.30 P.M. Induction was somewhat prolonged and difficult. He made attempts to sit up, and required a large amount of ether to obtain full anæsthesia. There was some cyanosis at this stage due to the patient holding his breath.

Shortly after full anæsthesia was obtained the breathing became shallow and the face cyanosed again, the pulse remaining of good volume and tension and the pupils contracted. The ether mask was removed and some carbon dioxide administered with the inspired air. The respirations at once became deep and quick and the colour excellent. There was no shock or hremorrhage during the operation. The anæsthetic was discontinued at 2.30 P.M., and the patient sent back to the ward. At 3.25 his condition was good except for some slight cyanosis. At 3.30 the face was more markedly cyanosed, the pulse fairly strong and regular, and the breathing stertorous, with sounds of mucus in the trachea. There was no other obstruction, as a Connell airway was in the mouth. At 3.35 the pulse became almost imperceptible, the breathing difficult, and cyanosis marked. Strychnine gr. 1/30, oil of camphor gr. $1 \frac{1}{2}$, were given hypodermically. Artificial respiration was begun, but a few minutes later the patient died. A post-mortem was done 19 hours after death. There was marked fatty degeneration of the myocardium with dilatation of the heart.

It is remarkable that more patients with this condition of the heart do not die upon the operating table. In this case the patient's attempt to sit up during induction of full anæesthesia and the cyanosis must have helped to overtax a heart only just able to function under normal conditions.

CASE 5.-An unmarried woman, aged 30, admitted to the Ross Memorial Pavilion as a patient of Dr. Hamilton White, to undergo an operation for deviated septum masi. She had always led an active life and had never noticed any special distress or disturbance referable to the heart or lungs, except a certain amount of shortness of breath on exertion at times during the preceding 10 or 12 months. Just before she entered the hospital she had been leading a particularly strenuous social life. The family history was negative and her previous health good, except for an attack of appendicitis for which she had been operated on some years before. The pulse was 72. Blood pressure 108-68; there was occasional arrhythmia. There was no cardiac enlargement, both sounds being well heard at the apex and fairly strong, but the second sounds at the pulmonary and aortic areas appeared rather weak. She was prepared for operation in the usual way. A hypodermic injection of morphine gr. 1/4 and atropine gr. $1 / 150$ were given at 7.40 on the moraing of the operation. Nitrous oxide gas and ether were used to induce anæsthesia, a few drops of chloroform being given just before the patient passed into the stage of full anzesthesia. The induction was perfectly smooth. The intratracheal catheter was introduced and the operation carried out. The anæsthetic was stopped at 9 A.M. The patient left the operating room in good condition.

An hour after return to her room she woke up, retched and vomited. Dr. S. H. Martin visited her at 12.10 P.M. and found the pulse somewhat weak and irregular, and recommended that she should be carefully watched. At 12.30 she was worse, the pulse was noticeably weaker and between 60 and 70 a minute. The face was greyish. At 12.50 the pulse conld not be felt at the wrist and the patient looked as if she were dying. The foot of the bed was elevated, caffeine hypodermically and rectal salines with whisky were given. After 20 minutes, the pulse began to come back and the condition to improve, so that by night the patient was fairly well. She left the hospital a few days later quite recovered. Before leaving she was examined by Dr. C. F. Moffat with the electrocardiograph. His report is as follows:-

"The ventricular complex is split and bizarre, kut not prolonged in the third lead. The $T$ wave third lead is inverted. There is a certain amount of sinus arrhythmia, otherwise the tracing is normal. From a consideration of the electrocardiogram there is evidence of , disturbed ventricular function, probably chronic in nature."

This case, like the second and fourth, showed how little warning the anæsthetist may get of trouble ahead by the examination of the heart. Examination with the electrocardiograph before operation would probably give us much more accurate information as to the risk to the patient.

THE SIGNIFICANCE OF

DISCHARGE FROM THE NIPPLE IN BREAST LESIONS.

By E. CATHERINE LEWIS, M.S. LoND., F.R.C.S. ENG.,

ASSISTANT SURGEON, ROVAT FREE HOSPITAI AND THE SOUTH LONDON HOSPITAY, FOR WOMEN.

THE series of cases on which the following article is based include all those admitted for lesions of the breast to the surgical wards of the Royal Free Hospital during the past ten years (1912-1921 inclusive). The specimens of breasts ( 44 in number) in the pathological museum have also been examined : many of these date from a much earlier period than those in the clinical series.

\section{Clinical Series.}

The cases in the clinical series number 251, of which 247 were female, 4 male; and among these there were only 26 patients, all of them women, who gave a history of discharge from the nipple. In these 26 cases the lesions were as follows: Spheroidal-celled carcinoma, 9 ; chronic mastitis (cystic and non-cystic), 6 ; duct carcinoma, 4; duct papilloma, 4 ; Paget's disease of the nipple, 1 ; abscess, 1 ; seborrhœic dermatitis of the nipple, 1 .

These figures cannot be considered alone, but must be taken in conjunction with the number of patients admitted for the respective lesions, as some of these are so much commoner than others. Thus, in the case of spheroidal-celled carcinoma the total number of patients treated in the ten-year series was 129 , giving a percentage of about 7 with discharge. The percentage in chronic mastitis was $13 \cdot 3$, in duct carcinoma $36 \cdot 3$, and in duct papilloma 100 .

Incidence in Different Varieties of Disease.

According to the figures in this series, therefore, the only disease in which discharge is constant is 
duct papilloma. On further inquiry into the history of these four patients it was found that in each case a small swelling was present in the sub-areolar region, which swelling on excision proved to be a dilated duct containing the small innocent growth. It is, perhaps, unfortunate for the purpose of analysis of these cases that such a swelling was found in each of these four, as, from time to time, a complaint of discharge is made by patients in whom, on examination. no other abnormality can be detected in the breast, but who are almost certainly suffering from this lesion. It remains possible that duct papillomata may exist in the more remote parts of the gland without giving any sign of their presence. On the other hand, there is one breast lesion which, apparently, never gives rise to nipple discharge: the fibro-adenoma in all its different varieties, including the mixed solid and cystic kind,

\section{Situation and Nature of the Lesion giving Rise to Discharge.}

If the appended diagrams are examined it will be seen that in each case where discharge was present. the lesion lay immediately beneath the nipple; and in the specimen of chronic mastitis which is not figured the breast was riddled with cysts, several of which were in the sub-areolar region. I made the same observation when looking into the histories of the patients in the clinical series; in every case where discharge had been complained of either the clinical examination or a diagram or a description of the parts removed at operation showed the lesion to be in close proximity to the nipple. Cases of duct carcinoma, the disease in which, above all others, one would expect a bloodstained discharge, did not manifest this symptom

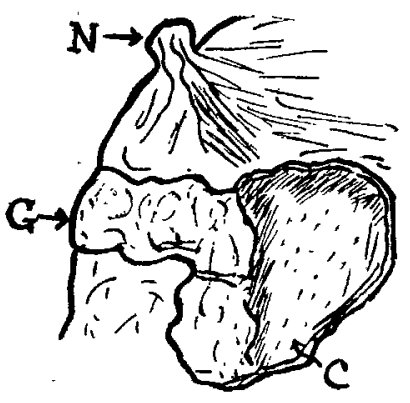

Discharge absent.
2

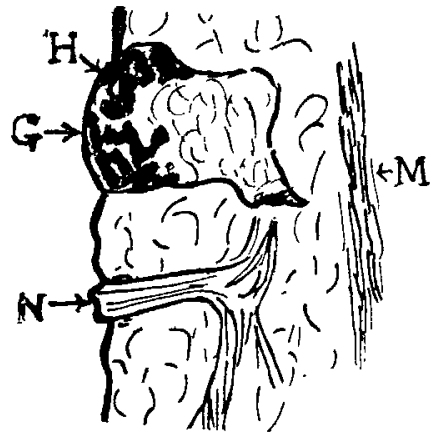

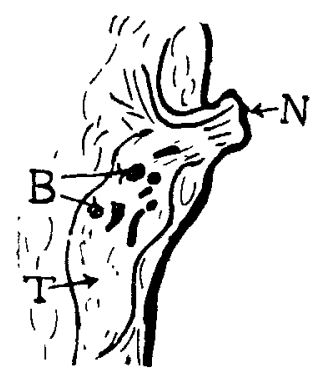

Discharge present.

Figs. 1, 2, 3, Duet carcinoma.

4

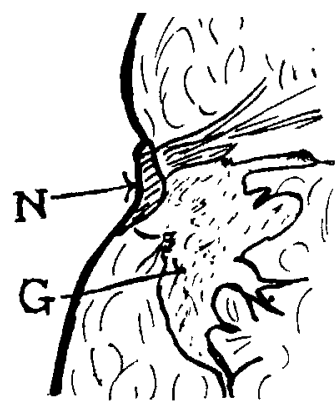

Discharge present.

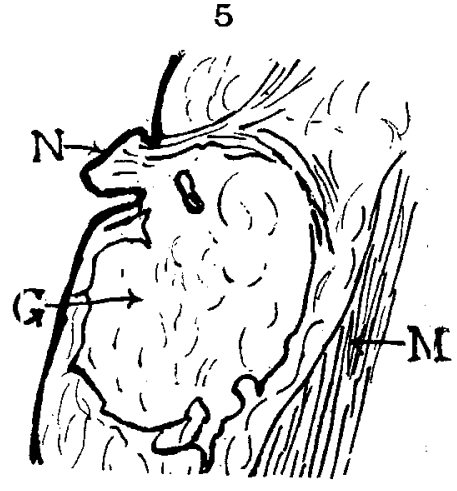

Figs. 4 and 5, Spheroidal-celled carcinoma.

$\mathbf{N}=$ Nipple. $\mathbf{G}$ and $\mathbf{T}=$ Growth involving skin. $\mathrm{C}=$ Cyatic degeneration in growth. $\mathrm{H}=\mathrm{H} \rightsquigarrow \mathrm{morrhage}$ into growth. $\mathbf{M}=\mathrm{Muscle}$. $\mathrm{B}=$ Dilated ducts containing blood-stained discharge.

formerly called Brodie's sero-cystic sarcoma. This is only reasonable when we remember that these are innocent tumours entirely contained within their own capsule, and which, even when large and situated immediately beneath the nipple, are readily shelled out from the surrounding tissues and can have no possible connexion with the ducts through which a secretion must pass to reach the surface of the nipple.

Of other breast lesions there was no history of discharge in the cases of tuberculous mastitis, galactocele, and one case of melanoma included in the series. In the first-named this may well be accidental. Further, in the cases with discharge we may pass over the two of disease of the nipple itself as presenting no difficulty in diagnosis, and also the single case (out of ten of the kind) of abscess, as here the discharge was demonstrated to be pus. We are left, then, in the clinical series with four varieties of lesion giving rise to discharge from the nipple : Carcinoma $(a)$ columnarcelled, $(b)$ spheroidal-celled, duct papilloma, and chronic mastitis. The discharge in all varieties was sometimes described as being blood-stained, in a few cases clear or blood-stained on different occasions, in the majority serous, either yellow or greenish. It was never profuse.

\section{Pathological Specimens.}

To turn now to the museum specimens that were available. Of the 44 cases a history of discharge was given in six-e.g., duct carcinoma, 1 (out of 4 specimens of this disease); spheroidal-celled carcinoma, 3 (out of 18 ); chronic mastitis with multiple cysts, 1 ; malignant disease of the nipple, 1 . In the single case of duct papilloma in the collection, in which the duct has dilated to the size of a small orange, the presence or absence of discharge is, unfortunately, not mentioned in the history. The appended diagrams (Figs. 1 to 5 ) which represent the slice of breast mounted in each jar, give a rough indication of the position of the lesion and its relation to the nipple. In this collection also we will ignore the case of epithelioma of the nipple, which would be self-evident, and confine our attention to the cases of malignant growth originating in the substance of the breast and those of chronic inflammation, unless the growth involved the sub-areolar region. It must be remarked that the converse does not hold good; many cases with tumours close to the areola and, indeed, several advanced ones involving the whole breast did not give this sign. In malignant cases the presence or absence of retraction of the nipple appears to have no bearing on the case ; neither does the rate of growth. Ige again does not appear to be a factor of importance.

One other point seemed to stand out. In all the malignant cases except one the patient had noticed the presence of a sucelling in the breast for some time previous to the appearance of the discharge; this fact is all the more significant when one remembers how frequently a patient suddenly becomes cognisant of a large tumour in the breast, whereas it is apparent to the surgeon that it must have been there for many months. On the other hand, a discharge from the nipple is not likely to pass unnoticed, and it seems reasonable to suppose that, in the one instance where it was recorded as being the first symptom, this was merely due to the patient's faulty powers of observation.

Chronic mastitis, whether of the solid or cystic variety, usually manifests itself in other ways, and it seems that the presence of discharge cannot help us to detect an early malignant change, as it may occur in either condition. I can, however, call to mind two or three cases in which nipple discharge was the only symptom, and in which clinical examination was absolutely negative except that pressure on the areola at one point, and one point only, elicited a drop of fluid, usually dark brown in colour. These patients, from one reason or another, refused operation, and presented exactly the same picture when re-examined several months later. These could only have been instances of a minute duct papilloma which, quite impalpable itself, had failed to cause sufficient obstruction to dilate the duct behind it. Papillomata only appear to occur in the larger ducts and not in the small ones towards the periphery of the gland; or it may be that they remain unrecognised in the latter situation. 
Bearing of the Discharge on Diagnosis and Treatment.

It seems fairly clear that the determining factor is the relation of the lesion to the nipple, and not the nature of the lesion itself; but for diagnosis we can divide the cases into two groups - that in which the nipple discharge is accompanied by one or more swellings in the breast, and that in which it is the sole symptom. Into the former class fall practically all the spheroidal-celled and duct carcinomata, and also the chronic inflammations with this sign, and some of the papillomata, and it does not seem as if the discharge itself is of any great value in distinguishing between them, as, although the percentage of duct carcinomata is highest, that of chronic mastitis next, and of spheroidal-celled cancers least, yet the number of the last-named is sufficiently large not to warrant their exclusion on the ground of improbability. In any case there is no particular value in making a clinical diagnosis between two varieties of carcinoma. In the various forms of chronic inflammation the characteristic knotted feel of the gland is usually a safe guide, and a small globular swelling in the region of the areola is highly suggestive of a duct obstructed by a papilloma. These are not the difficult cases. It is in the second group, where discharge is the only abnormality that can be detected, that doubt as to the proper diagnosis and line of treatment may occur. The question of malignancy inevitably arises in any region where unexplained bleeding occurs, and some authorities go so far as to say that the breast should be amputated on this sign alone. This drastic suggestion is apt not to commend itself to a patient who feels that she is only complaining of a trivial matter ; as the result of it, she may elect to do without treatment of any kind-a dangerous decision. If the surgeon feels really suspicious of the presence of a malignant growth radical operation should, of course, be strongly urged, but it is the object of this paper to show that discharge, whether blood-stained or clear, from the nipple of an otherwise apparently normal breast is in all probability not due to a malignant growth, and is, therefore, not an indication for immediate and complete amputation. The reasonable procedure would seem to be to determine the direction from which the discharge comes, and to excise that portion of the areola and neighbouring tissues. The piece removed should be subjected at once to a thorough naked-eye examination (a frozen section might be lucky, but might well miss the lesion altogether). Should the appearance of the tissues be suspicious the major operation must be proceeded with, but if not, if a discrete papillomatous growth is discovered or if no lesion at all is found, nothing further need be done. The patient should, of course, be kept under observation.

To sum up-I do not wish for a moment to suggest that a surgeon should hesitate to amputate a breast when there is the smallest suspicion of malignancy, nor to advocate the dangerous procedure of double operation with intermediate microscopic examination of the tissue removed in the first instance, but to suggest that discharge from the nipple is not in itself a definite expression of malignancy and should not be treated as such unless the suspicion is strengthened by the rest of the history and clinical picture.

L I T E R A R Y I N T EL I I G E C E.-Messrs. George Routledge and Sons are preparing a series of volumes on chemistry designed for the use of post-graduate readers and chemistry designed for the use of post-graduate readers and
others. The Series is under the editorship of Sir William A. Tilden, F.R.S., and Prof. J. C. Philip, F.R.S., and will include "The Metastability of Matter," by Prof. Ernest Cohen " Oxidation and Reduction in Organic Chemistry," by Dr. Oscar L. Brady, D.Sc.; "Physical Aspects of Organic Chemistry," by Prof. T. M. Lowry, F.R.S. ; "Atomic and Molecular structure in Relation to Properties," by Dr. Mroecular Structure in Relation to Properties," by Dr; by Prof. J. R. Partington, D.Sc.; "Space Formulæ in Carbon Compounds," by Prof. Jo elyn F. Thorpe, F.R.S., Ingold, B.Sc. and its Practical Application," by Prof. Henry Bassett.

\section{Clinital 影otes:}

\section{MEDICAL, SURGICAL, OBSTETRICAL, AND THERAPEUTICAL.}

A CASE OF

\section{A NTRO-CHOANAL POLYPUS OF EXCEPTIONAL SIZE.}

By G. W. Dawson, F.R.C.S. IREL.,

SURGEON, THROAT HOSPITAI, GOLDEN SQUARE, LONDON.

THE accompanying illustration is of an exceptionally large antro-choanal polypus, weighing $9 \frac{1}{2}$ drachms, occurring in a man 47 years of age, who was content to endure its presence for several years. It is a globular, glistening, pearl-coloured, firm growth, apparently filling up the entire pharynx, and bulging the soft palate forward, the uvula being spread out on its surface ; its lower limit can only be seen with difficulty by strongly depressing the tongue, when it can be seen to reach close to the entrance to the larvnx. It is remarkable how little respiration or deglutition is impeded. The subject can breathe through either nostril quite well. Bevond the peculiar muffled speech, and the occurrence of fits of suffocation should he happen to turn on his back during sleep, there are no symptoms.

Examination. - Anteriorly the nose appears normal, it, is only on the application of cocaine that the stalk of the growth can be demonstrated, lying under the anterior end of the middle turbinate bone. Transillumination was much darker on the affected side, and by passing the finger into the post-nasal space the cord-like stalk was easily felt to disappear into the right choana.

Operation.--The antrum was opened through the canine fossa, the attachment was close to the orifice of the cavity, the lining membrane of which was in a state of polypoid degeneration.

Pathology of the Condition.-The growth originates by a cystic or polypoid degeneration of the lining membrane of the antrum, close to its orifice. It may be laid down as an axiom that a polypus growing from a cavity with a narrow opening - whether it is the antrum, the sphenoidal sinus, or an ethmoidal cell-is always single, it being impossible for another polypus to pass through the blocked opening. In recent cases the surface is covered with ciliated epithelium which in places is squamous owing to pressure. The body is made up of a network of fibrous tissue, forming spaces which are filled with the mucigenous watery material. There is, therefore, no true cyst present. In long-standing cases with years of growth the latter presents a solid firm appearance, due for the most part to its many covering layers (six or seven) of epithelium flattened on the surface, but more cubical in the deeper layers, and there is more fibrous tissue in the body of the growth.

Treatment.-After the application of cocaine, the polypus may be removed by passing a snare or a Luc's forceps through the anterior nares. Sometimes the pedicle as it passes backwards under the middle turbinate may be engaged by a blunt hook, traction on which will tear away the growth at its origin. The simplest of all to deal with are those that appear below the palate-it is only necessary to raise the latter and seize the polypus with a luc's forceps, when the whole growth will come arvay at its origin in the antrum. In many cases these simple measures suffice. Where trans- 